Physics Communications

Elsevier Editorial System(tm) for Computer

Manuscript Draft

Manuscript Number: CPC-D-16-00013R1

Title: An efficient and portable SIMD algorithm for charge/current deposition in Particle-In-Cell codes

Article Type: Computer programs in physics paper

Keywords: Particle-In-Cell method, Message Passing Interface, OpenMP, SIMD Vectorization, AVX, AVX2, AVX-512, Tiling, Multi-core architectures, Many-Integrated Core (MIC) architectures, x86 architectures

Corresponding Author: Dr. Henri Paul VINCENTI, Ph. D

Corresponding Author's Institution: Lawrence Berkeley National Laboratory

First Author: Henri Paul VINCENTI, Ph. D

Order of Authors: Henri Paul VINCENTI, Ph. D; Mathieu Lobet, PhD; Remi Lehe, PhD; Ruchira Sasanka, PhD; Jean-Luc Vay, PhD

Abstract: In current computer architectures, data movement (from die to network) is by far the most energy consuming part of an algorithm (\$ \approx 20\$pJ/word on-die to \$lapprox 10,000\$pJ/word on the network). To increase memory locality at the hardware level and reduce energy consumption related to data movement, future exascale computers tend to use many-core processors on each compute nodes that will have a reduced clock speed to allow for efficient cooling. To compensate for frequency decrease, machine vendors are making use of long SIMD instruction registers that are able to process multiple data with one arithmetic operator in one clock cycle. SIMD register length is expected to double every four years. As a consequence, Particle-In-Cell (PIC) codes will have to achieve good vectorization to fully take advantage of these upcoming architectures. In this paper, we present a new algorithm that allows for efficient and portable SIMD vectorization of current/charge deposition routines that are, along with the field gathering routines, among the most time consuming parts of the PIC algorithm. Our new algorithm uses a particular data structure that takes into account memory alignement constraints and avoids gather/scatter instructions that can significantly affect vectorization performances on current CPUs. The new algorithm was successfully implemented in the 3D skeleton PIC Code PICSAR and tested on Haswell Xeon processors (AVX2-256 bits wide data registers). Results show a factor of $\$$ \times $2 \$$ to $\$ \backslash$ times $2.5 \$$ speed-up in double precision for particle shape factor of order $\$ 1 \$$ to $\$ 3 \$$. The new algorithm can be applied as is on future KNL (Knights Landing) architectures that will include AVX-512 instruction sets with 512 bits register lengths (8 doubles/16 singles). 
Dear Editor,

Please find attached our complete revision of our paper (revised version of the manuscript along with detailed answer to reviewers). We apologize for the delay, which is mainly due to upgrade of the cluster on which we performed our tests.

As you requested, we removed the pages of the code in appendix and submitted the paper as a computer program in physics. We added a Program summary in the manuscript and submitted the source files containing subroutines detailed in the paper.

Sincerely yours,

\section{Henri VINCENTI}

Marie Curie Research Fellow

Accelerator Technology and Applied Physics Division

Lawrence Berkeley National Laboratory - Building 71, Mailstop 259

Berkeley, CA 94720, USA

Tel: (1) 415-706-8144 - Email: hvincenti@lbl.gov 


\section{Answer to reviewer \#1:}

We thank reviewer \#1 for his careful reading of our manuscript and for all the useful comments he made on the paper. Below are answers to his/her comments:

- Comment \#1:

"1. What is the relation between the $10 \mathrm{pJ} /$ word which can be read in the abstract as a feature of the present computers and the Table 1 where such number (24 pJ) can be read only for 2019 year?"

- Answer \#1:

In the abstract we initially just gave orders of magnitude but for better coherence with the main text we took into account your comment by modifying the first sentence of the abstract (changes are highlighted with bold font):

"In current computer architectures, data movement (from die to network) is by far the most energy consuming part of an algorithm (\$ |approx 20\$pJ/word on-die to $\$$ |approx 10,000\$pJ/word on the network)."

- Comment \#2:

"2. In Table 1 caption, reference [5] can be read while Ref [2] to [4] did not appear year. The reference number should appear in the text by increasing order."

- Answer \#2:

We corrected this and references now appear in increasing order in the main text.

- Comment \#3:

"3. For the test reported on a plasma in Table 2, the dimensionless number thermal velocity $x$ mesh size/time step should be provided to assess the typical number of particle which exit a cell at every time step."

- Answer \#3:

We took your comment into account by replacing "plasma temperature" by "thermal velocity" and estimating the total number of particles leaving a cell after each time step:

- Page 5: "Particles are randomly distributed on the grid and have a thermal velocity of $\$ v_{-}\{t h\}=\mid$ approx $0.1 c \$$ with $\$ c \$$ being the speed of light in vacuum. For a time 
step imposed by the Courant condition of the Maxwell solver $\$ d t=0.57 d x / c \$$ (where $\$ d x \$$ is the mesh size) and $\$ n_{-} p=10$ particle per cells, the total number of particles leaving a cell after one time step is $\$ n_{-} p \mid$ times $v_{-}\{t h\} \mid$ times $d t / d x \mid$ approx 0.57\$. After 20 time steps, nearly all particles have left their original cell. "

- Comment \#4:

"4. Can we deduce from comments read in Table 2 captions that the tests are operated within shared memory context only?"

- Answer \#4:

You are right; the tests were performed on a single socket of the Edison cluster at NERSC. This is emphasized by the following sentence in the main text:

"The tests were performed on one MPI process and a single socket, on the Edison cluster at NERSC."

- Comment \#5:

"5. In Sec. 5, Fig. 4 suggest the computer time gain as a function of the number of particle per cell $N_{-}$c. Have the authors some scaling law gain $\left(N_{-} c\right)$ to propose? A third case in addition to $N_{-} c=10$ and 40 should be useful."

- Answer \#5:

To address your comment, we modified Fig. 4 and 5 of the paper and added another panel with the evolution of speed-up gains with the number of particles per cells. As the cluster was down several times lately due to upgrade with future Knights Landing, this part took us longer than we initially expected. Moreover, we slightly modified our ways of computing speed-ups and these are now estimated on a higher numbers of iterations, which very slightly changes figures from the original draft. 


\section{Answer to reviewer \#2:}

We thank reviewer \#2 for his careful reading of our manuscript and for all the useful comments he made on the paper. Below are answers to his/her comments:

- Comment \#1:

"Abstract: While the "fat node" argument is clearly true, it seems quite wordy for an abstract. Since it is widely accepted that vectorisation is one of the key performance optimisations required for modern HPC hardware I would recommend to shorten the first 6 lines a little."

- Answer \#1:

We addressed your comments and reformulated some of the first lines of the abstract. Besides, we removed "fat nodes" in the abstract. New abstract is detailed below:

"In current computer architectures, data movement (from die to network) is by far the most energy consuming part of an algorithm (\$1approx 20\$pJ/word on-die to $\$$ |approx 10,000\$pJ/word on the network). To increase memory locality at the hardware level and reduce energy consumption related to data movement, future exascale computers tend to use many-core processors on each compute nodes that will have a reduced clock speed to allow for efficient cooling. To compensate for frequency decrease, machine vendors are making use of long SIMD instruction registers that are able to process multiple data with one arithmetic operator in one clock cycle. SIMD register length is expected to double every four years."

- Comment \#2:

"a) Is the data structure difference to reference [3] a simple Array-of-Structure (AoS) vs. Structure-of-Array (SoA) issue? If so, please clearly state this, since it seems crucial."

- Answer \#2:

In ref [3], Decyk et al use a Fortran array ppart(1 : ndims, $1:$ nppmax,1 : ntiles) for particles where ndims is the number of particle propertie (position, velocity, weight for instance), nppmax the maximum number of particles and ntiles the number of tiles. The main problem using this structure is related to memory usage. Indeed, if one tile has many more particles than other tiles, then nppmax will have to be set to this number for all tiles potentially leading to "out of memory" errors when the 
number of tiles is high. Moreover, if nppmax needs to be increased at runtime, it will eventually lead to a deallocation and reallocation of the whole array ppart.

To avoid costly reallocations and out of memory errors, we instead use Fortran derived types. Instead of having a regular Fortran 3D Array ppart, we define a Fotran array of tiles array_of_tiles, each "tile" being a Fortran derived type containing particle arrays (positions, velocities, weights). With this data structure, two tiles can have a different number of particles. Moreover, each tile can be resized indepentently at runtime.

In short, there is no much difference in terms of data layout between our data structure and the one used in [3] but our implementation allows for much better memory management.

- Comment \#3:

"b) The performance increase of $3 x$ is already quite impressive and tiling clearly complements the caching optimisation in this paper, but it seems unclear just how much of the overall performance increase is due to increased cache locality from tiling and how much is due to vectorisation. Further context, such as rooflines and more detailed performance metrics could be used to make thisclearer in this section and section $5 . . "$

- $\quad$ Answer \#3:

The speed-up $S_{1}$ due to tiling only is presented on Table 2. On Fig. 4 and Fig5., we only reported speed-up $S_{2}$ due to vectorization. The total speed-up is $S=S_{1}{ }^{*} S_{2}$.

To address your comment and clearly highlight this we changed:

1. the caption title of Figs 4 and Figs 5 to "Speed-up brought by vectorization with the new charge deposition algorithm on Cori" and "Speed-up brought by vectorization with the new current deposition algorithm on Cori". We also changed the title of the benchmarks section to "Speed-up brought by the new vector algorithm on Cori".

2. We added this sentence on page 13: "If speed-ups from particle tiling (\$3|times\$) and from vectorization (up to \$2.7|times\$ for charge deposition at order \$1\$) are combined, we can gain a cumulated speed-up of up to \$8|times\$ for deposition routines."

We understand that roofline curves would be a plus but these would imply a larger modification of the paper. Since the submission of this paper, we performed extensive roofline performance tests on both Haswell and KNL and these results will be presented soon in another paper. 
- Comment \#4:

"As a consequence, particle exchanges between tiles account in practice for a very small percentage of the total PIC loop (a few percents)." This sounds quite vague. Have you run this with varying particle densities; does this affect results? »

\section{- Answer \#4:}

The timing of the different routines of the PIC algorithm shows that particle exchange between tiles only accounts for less than $4-5 \%$ of the total computation time. All the runs are performed with a thermalized plasma. The number of particles exchanged at each time step depends on the number of pseudo-particle per cells and thermal velocity (see Answer \#3 to Reviewer \#1). In our benchmarks, we used a rather high thermal velocity $(0.1 \mathrm{c})$ and we varied the number of particles per cell. In each case, we did not see any performance bottlenecks due to particle exchange between tiles.

One major performance issue that we did not discuss here is the parallelization of particle exchanges between tiles. This will be published in another paper. To address this, we found an efficient way of distributing tiles to threads in a "double chessboard" pattern. This ensures that two different threads handling two different tiles cannot exchange particles with the same adjacent tile (therefore avoiding contention between threads).

- Comment \#5:

"* Listing 3: A missing space in line 18.»

- Answer \#5:

This has been fixed.

- Comment \#6:

"* Section 4.1.2:a) The introduction of the RHOCELLS data structure essentially replicates data. Since memory-per-core ratios are generally decreasing, how does this affect the memory footprint of the algorithm? And also, assuming varying cache sizes across HPC architectures, is it just a matter of adjusting tile size to keep RHOCELLS in cache?»

- Answer \#6:

You are right, there is a slight memory overhead with our technique. There are 8grid nodes per cells in 3D and we only replicate grid nodes shared by neighboring cells. 
However, rhocells can be allocated and deallocated by each thread at runtime (each thread handles one tile at a time). As there are always much fewer threads than tiles, the total memory footprint at runtime is rather low.

Furthermore, as you highlighted in your comment, the tile size can be adjusted depending on the architecture so that rhocells fit in cache.

- Comment \#7:

" b) You have hoisted the particle weights and are using a generic indexing formula into RHOCELLS. Could this be done without the RHOCELLS buffer and would you expect it to still increase performance? »

- Answer \#7:

Unfortunately not because rhocells allows avoiding a lot of gather/scatter that still significantly hinder vectorization performances. In that case, you would achieve near sequential performance with the regular array rho except if particles were sorted onto the grid. The key of this new data structure is precisely to avoid gather/scatter in the particle loop. We still get gather/scatter when reducing rhocells in the regular array rho but the number of gather/scatter are now proportional to the number of cells this time. As in the PIC algorithm we usually use much more particles than cells, we can get significant performances from this technique.

- Comment \#8:

“c) Reference to line 39 in listing 3 is off. It should probably be line 41-42.»

- Answer \#8:

This has now been fixed.

- Comment \#9:

"* Section 4.2: Could padding be used here to enforce better alignment for the order 2 shape, or would the resulting overhead be too large? Also, why are you focusing on L1 cache size and not L2?»

- Answer \#9:

Padding could be used to ensure 64 bytes memory alignment (as required for KNL architectures for instance) of two different cells in rhocells(8,Ncells) but this would have an extra memory cost that we did not study here. You are right, we changed the following sentence "are not in L1 cache " to " are not in cache". 
- Comment \#10:

"*Section 5:a) As mentioned above, how important is it to force the RHOCELLS data structure into cache, and how does the tile size affect the vectorisation results? What is the penalty for missing the tiling sweet-spot? Would a sweep of tile sizes add to the information conveyed in the paper?

- Answer \#10:

Vector performances will be measurable only if the code is not memory bound (i.e the algorithm have a rather high flop/byte ratio). If data are not in cache then as highlighted in the introduction section of this paper, vector performances are not visible at all as the majority of the execution time is spent in doing memory exchanges and not computations. As you highlight in your comment, there definitely is a tiling "sweet spot". The tile size has to fit in L2 cache in order to see improvements with tiling. If tile is bigger than L2 cache size, performance drops very quickly. We measured this on different architectures. For architectures with no L3 cache (e.g intel KNC), the speed-up gain due to tiling can be as high as 7 on the whole code.

If tile size is smaller than L2, performances slowly decrease with the tile size. These results will also be detailed in a more comprehensive paper dedicated to the high performance optimizations done in the PICSAR kernel.

- Comment \#11:

" b) There is no indication of the overall performance limitations of the algorithm. Would a roofline plot be useful in illustrating how effective your optimisations are? »

- Answer \#11:

This is now clearer with the modifications of Fig. 4 and Fig. 5 (asked by reviewer \#1) where we plotted the speed-up gain of the vector algorithm as a function of the number of particles per cell. The algorithm will perform efficiently as soon as there are more particles than cells. We agree with you that rooflines plots would be really great to assess the flop/byte ratio of the algorithm and the performance of the vector algorithm for this flop/byte ratio. However, as mentioned in answer \#3, this would imply large modifications of the paper at this stage. All our roofline studies on the whole PICSAR kernel on intel Haswell and KNL architectures will be submitted soon.

- Comment \#12: 
"Conclusion: I believe your point about avoiding gathers and how to do it is a very important one. I think a clear statement of why this algorithm is effective would improve this section, ie. something like: "The algorithm uses an additional data structure to store the deposition values in cache and thus alleviate the effect of costly memory gather operations."»

- Answer \#12:

We addressed your comment and modified the text of the paper as follows:

"This method uses a new data structure for grid arrays (charge/currents) ensuring data alignement and contiguity in memory which are essential to avoid many gather/scatter operations that can significantly hinder vector performances on modern architectures." 


\title{
An EFFICIENT AND PORTABLE SIMD ALGORITHM FOR CHARGE/CURRENT DePosition in PARTiCle-IN-CELl CODES
}

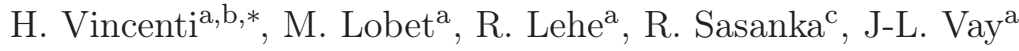 \\ ${ }^{a}$ Lawrence Berkeley National Laboratory, 1 cyclotron road, Berkeley, California, USA \\ ${ }^{b}$ Lasers Interactions and Dynamics Laboratory (LIDyL), Commissariat $\tilde{A}$ l'Energie Atomique, Gif-Sur-Yvette, France \\ ${ }^{c}$ Intel corporation, Oregon, USA
}

\begin{abstract}
In current computer architectures, data movement (from die to network) is by far the most energy consuming part of an algorithm $(\approx 20 \mathrm{pJ} /$ word on-die to $\approx 10,000 \mathrm{pJ} /$ word on the network $)$. To increase memory locality at the hardware level and reduce energy consumption related to data movement, future exascale computers tend to use many-core processors on each compute nodes that will have a reduced clock speed to allow for efficient cooling. To compensate for frequency decrease, machine vendors are making use of long SIMD instruction registers that are able to process multiple data with one arithmetic operator in one clock cycle. SIMD register length is expected to double every four years. As a consequence, Particle-In-Cell (PIC) codes will have to achieve good vectorization to fully take advantage of these upcoming architectures. In this paper, we present a new algorithm that allows for efficient and portable SIMD vectorization of current/charge deposition routines that are, along with the field gathering routines, among the most time consuming parts of the PIC algorithm. Our new algorithm uses a particular data structure that takes into account memory alignement constraints and avoids gather/scatter instructions that can significantly affect vectorization performances on current CPUs. The new algorithm was successfully implemented in the 3D skeleton PIC code PICSAR and tested on Haswell Xeon processors (AVX2-256 bits wide data registers). Results show a factor of $\times 2$ to $\times 2.5$ speed-up in double precision for particle shape factor of order 1 to 3 . The new algorithm can be applied as is on future KNL (Knights Landing) architectures that will include AVX-512 instruction sets with 512 bits register lengths (8 doubles/16 singles).
\end{abstract}

Keywords: Particle-In-Cell method, Message Passing Interface, OpenMP, SIMD Vectorization, AVX, AVX2, AVX-512, Tiling, Multi-core architectures, Many-Integrated Core (MIC) architectures, x86 architectures

\section{PROGRAM SUMMARY}

Manuscript Title:An efficient and portable SIMD algorithm for charge/current deposition in Particle-In-Cell codes Authors: H. Vincenti, M. Lobet, R. Lehe, R. Sasanka, J-L Vay

Program Title: vec_deposition

Journal Reference:

Catalogue identifier:

Licensing provisions: "none"

Programming language: Fortran 90

Computer:CPUs with SIMD vector units

Operating system: UNIX, WINDOWS. Can be compiled on any platform where a Fortran compiler is provided with OpenMP 4.0

Keywords: Particle-In-Cell method, Current/charge deposition, SIMD Vectorization, AVX, AVX2, AVX-512, OpenMP 4.0

Classification: 19. Plasma physics -19.8 kinetic models

\footnotetext{
* Corresponding author

Email addresses: hvincenti@lbl.gov (H. Vincenti), mlobet@lbl.gov (M. Lobet), rlehe@lbl.gov (R. Lehe), ruchira.sasanka@intel.com (R. Sasanka), jlvay@lbl.gov (J-L. Vay)
} 
External routines/libraries: OpenMP $>4.0$

Nature of problem:

Exascale architectures will have many-core processors per node with long vector data registers capable of performing one single instruction on multiple data during one clock cycle. Data register lengths are expected to double every four years and this pushes for new portable solutions for efficiently vectorizing Particle-In-Cell codes on these future many-core architectures. One of the main hotspot routines of the PIC algorithm is the current/charge deposition for which there is no efficient and portable vector algorithm.

Solution method:

Here we provide an efficient and portable vector algorithm of current/charge deposition routines that uses a new data structure, which significantly reduces gather/scatter operations. Vectorization is controlled using OpenMP 4.0 compiler directives for vectorization which ensures portability across different architectures.

\section{Restrictions:}

Here we don't provide the full PIC algorithm with an executable but only vector routines for current/charge deposition. These scalar/vector routines can be used as library routines in your 3D Particle-In-Cell code. However, to get the best performances out of vector routines you have to satisfy the two following requirements: 1) Your code should implement particle tiling (as explained in the manuscript) to allow for maximized cache reuse and reduce memory accesses that can hinder vector performances. The routines can be used directly on each particle tile. 2) You should compile your code with a Fortran 90 compiler (e.g intel, gnu or cray) and provide proper alignement flags and compiler alignement directives (more details in README file). 


\section{Introduction}

\subsection{Challenges for porting PIC codes on exascale architectures: importance of vectorization}

Achieving exascale computing facilities in the next decade will be a great challenge in terms of energy consumption and will imply hardware and software developments that directly impact our way of implementing PIC codes [1].

\begin{tabular}{|c|c|c|}
\hline Operation & Energy cost & Year \\
\hline DP FMADD flop & $11 p J$ & 2019 \\
\hline Cross-die per word access & $24 p J$ & 2019 \\
\hline DP DRAM read to register & $4800 p J$ & 2015 \\
\hline DP word transmit to neighbour & $7500 p J$ & 2015 \\
\hline DP word transmit across system & $9000 p J$ & 2015 \\
\hline
\end{tabular}

Table 1: Energy consumption of different operations taken from [2]. The die hereby refers to the integrated circuit board made of semi-conductor materials that usually holds the functional units and fast memories (first levels of cache). This table shows the energy required to achieve different operations on current (Year 2015) and future (Year 2019) computer architectures. DP stands for Double Precision, FMADD for Fused Multiply ADD and DRAM for Dynamic Random Access Memory.

Table 1 shows the energy required to perform different operations ranging from arithmetic operations (fused multiply add or FMADD) to on-die memory/DRAM/Socket/Network memory accesses. As 1pJ/flop/s is equivalent to $1 \mathrm{MW}$ for exascale machines delivering 1 exaflop $\left(10^{18} \mathrm{flops} / \mathrm{sec}\right)$, this simple table shows that as we go off the die, the cost of memory accesses and data movement becomes prohibitive and much more important than simple arithmetic operations. In addition to this energy limitation, the draconian reduction in power/flop and per byte will make data movement less reliable and more sensitive to noise, which also push towards an increase in data locality in our applications.

At the hardware level, part of this problem of memory locality was progressively adressed in the past few years by limiting costly network communications and grouping more computing ressources that share the same memory ("fat nodes"). However, partly due to cooling issues, grouping more and more of these computing units will imply a reduction of their clock speed. To compensate for the reduction of computing power due to clock speed, future CPUs will have much wider data registers that can process or "vectorize" multiple data in a single clock cycle (Single Instruction Multiple Data or SIMD).

At the software level, programmers will need to modify algorithms so that they achieve both memory locality and efficient vectorization to fully exploit the potential of future exascale computing architectures.

\subsection{Need for portable vectorized routines}

In a standard PIC code, the most time consuming routines are current/charge deposition from particles to the grid and field gathering from the grid to particles. These two operations usually account for more than $80 \%$ of the execution time. Several portable deposition algorithms were developed and successfully implemented on past generations' vector machines (e.g. CRAY, NEC) [3, 4, 5, 6, 7]. However, these algorithms do not give good performance on current SIMD architectures, that have new constraints in terms of memory alignement and data layout in memory.

To the authors' knowledge, most of the vector deposition routines proposed in contemporary PIC codes use compiler based directives or even $\mathrm{C}++$ Intel intrinsics in the particular case of the Intel compiler, to increase vectorization efficiency (e.g. [8]). However, these solutions are not portable and require code rewriting for each new architecture.

\subsection{Paper outline}

In this paper, we propose a portable algorithm for the direct deposition of current or charge from macro particles onto a grid, which gives good performances on SIMD machines. The paper is divided into four parts: 


\section{The PICSAR-EM3D PIC kernel}

PICSAR-EM3D is a standalone "skeleton" PIC kernel written in Fortran 90 that was built using the main electromagnetic PIC routines (current deposition, particle pusher, field gathering, Yee field solver) of the framework WARP [10]. As WARP is a complex mix of Fortran 90, C and Python, PICSAR-EM3D provides an essential testbed for exploring PIC codes algorithms with multi-level parallelism for emerging and future exascale architectures. All the high performance carpentry and data structures in the code have been redesigned for best performance on emerging architectures, and tested on NERSC supercomputers (CRAY XC30 Edison and testbed with Intel Knight's Corner coprocessors Babbage).

\subsection{PIC algorithm}

PICSAR-EM3D contains the essential features of the standard explicit electromagnetic PIC main loop:

(i) Maxwell solver using arbitrary order finite-difference scheme (staggered or centered),

(ii) Field gathering routines including high-order particle shape factors (order 1 - CIC, order 2 - TSC and order 3 - QSP),

(iii) Boris particle pusher,

(iv) Most common types of current depositions : Morse-Nielson deposition [1] (also known as direct $\rho \mathbf{v}$ current deposition) and Esirkepov [11] (charge conserving) schemes. The current and charge deposition routines support high-order particle shape factors (1 to 3 ).

\subsection{High performance features}

Many high performance features have already been included in PICSAR-EM3D. In the following, we give a quick overview of the main improvements that brought significant speed-up of the code and that are of interest for the remainder of this paper. A more comprehensive description of the code and its performances will be presented in another paper.

\subsubsection{Particle tiling for memory locality}

Field gathering (interpolation of field values from the grid to particle positions) and current/charge deposition (deposition of particle quantities to adjacent grid nodes) account for more than $80 \%$ of the total execution time of the code. In the deposition routines for instance, the code loops over all particles and deposit their charges/currents on the grid.

One major bottleneck that might arise in these routines and can significantly affect overall performance is cache reuse.

Indeed, at the beginning of the simulations (cf. Fig. 1 (a)) particles are typically ordered along the "fast" axis ("sorted case") that corresponds to parts of the grid that are contiguously located in memory. As the code loops over particles, it will thus access contiguous grid portions in memory from one particle to another and efficiently reuse cache.

However, as time evolves, the particle distribution often becomes increasingly random, leading to numerous cache misses in the deposition/gathering routines (cf. Fig. 1 (b)). This results in a considerable decrease 
(a) $t=0$, ordered



(b) $\mathrm{t}>0$, random

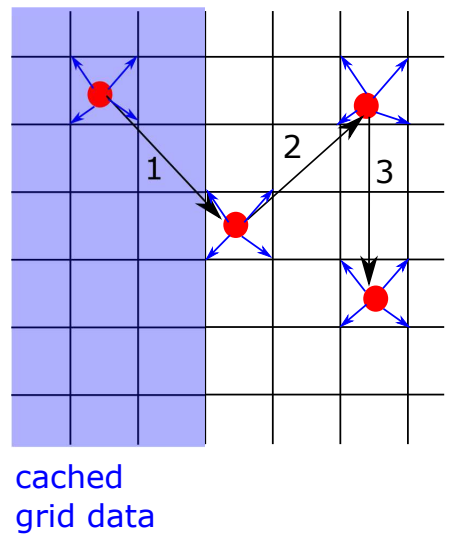

Figure 1: Importance of cache reuse in deposition routines. Illustration is given in 2D geometry for clarity, with CIC (linear) particle shapes. Panel (a) shows a typical layout at initialization $(\mathrm{t}=0)$ where particles are ordered along the "fast" axis of the grid, corresponding to grid cells (blue area) that are contiguous in memory. The loop on particles is illustrated with arrows and index of the loop with numbers 1 to 3 . Using direct deposition, each particle (red point) deposits (blue arrows) its charge/current to the nearest vertices ( 4 in $2 \mathrm{D}$ and 8 in $3 \mathrm{D}$ for CIC particle shapes). Panel (b) illustrates the random case (at $\mathrm{t}>0$ ) where particles are randomly distributed on the grid. As the algorithms loops over particles, it often requires access to uncached grid data, which then results in a substantial number of cache misses.

in performance. In 2D geometry, one MPI subdomain usually fits in L2 cache (256kB to $512 \mathrm{kB}$ per core) but for 3D problems with MPI subdomains handling 100x100x100 grid points, one MPI subdomain does not fit in cache anymore and random particle distribution of particles can lead to performance bottlenecks.

baseline PIC code (a)

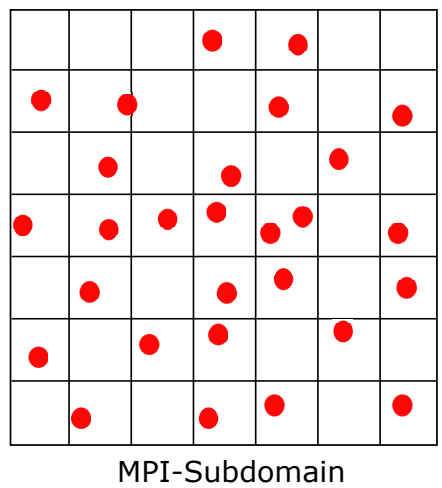

Particle tiling

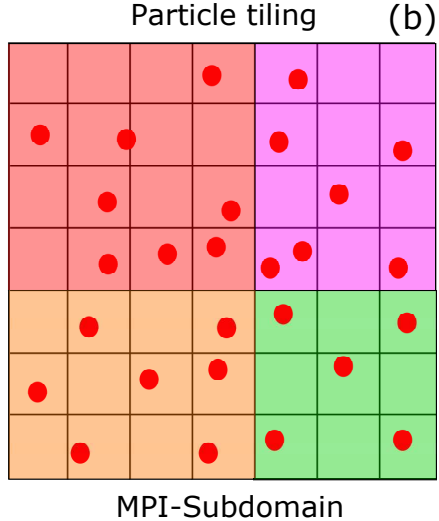

Figure 2: Particle tiling for efficient cache reuse. Panel (a) shows the usual configuration used in standard codes. There is one big array for particles for each MPI subdomain. Panel (b) shows the data structure used in PICSAR. Particles are grouped in tiles that fit in cache, allowing for efficient cache reuse during deposition/gathering routines.

To solve this problem and achieve good memory locality, we implemented particle tiling in PICSAREM3D. Particles are placed in tiles that fit in cache (cf. Fig. 1 (b)). In the code, a tile is represented by a structure of array Type(particle_tile) that contains arrays of particle quantities (positions, velocity and weight). All the tiles are represented by a 3D Fortran array array_of_tiles(:,:,:) of type particle_tile in the code. Our data structure is thus very different from the one in [12] which uses one large Fortran ppart(1: ndims,1: nppmax,1 : ntiles) array for all particles and tiles, where ndims is the number of particle attributes (e.g positions $x, y, z$ ), nppmax the maximum number of particles in a tile and ntiles the number of tiles. There are two reasons behind our choice:

(i) if one tile has much more particles than others, we considerably save memory by using our derived type compared to the array ppart. Indeed, in the latter case, if one tile has much more particles $n p$ than others, we would still need to choose nppmax $\geqslant n p$ for all the tiles, 
(ii) any tile can be resized as needed independently, without the need for reallocating the entire array of tiles.

Performance improvements of the whole code are reported on table 2 for tests performed on Intel Ivy Bridge (Cray XC30 Edison machine at NERSC). These tests show a speed-up of $\mathrm{x} 3$ in case of a random particle distribution. Cache reuse using tiling reaches $99 \%$. The optimal tile size ranges empirically between $8 \times 8 \times 8$ cells to $10 \times 10 \times 10$ cells. As will be shown later in the paper, having good cache reuse is crucial to increasing the flop/byte ratio of the proposed algorithm and obtaining improvements using vectorization.

\begin{tabular}{|c|c|c|}
\hline Tile size & Speed-up & L1 and L2 Cache reuse \\
\hline $1 \times 1 \times 1$ & $\times 1$ & $85 \%$ \\
\hline $10 \times 10 \times 10$ & $\times 3$ & $99 \%$ \\
\hline
\end{tabular}

Table 2: Speed-up of the whole PIC code brought by particle tiling. Tests were performed using a 100x100x100 grid with 10 particle per cells. Particles are randomly distributed on the grid and have a thermal velocity of $v_{t h}=\approx 0.1 c$ with $c$ being the speed of light in vacuum. For a time step imposed by the Courant condition of the Maxwell solver $d t=0.57 d x / c$ (where $d x$ is the mesh size) and $n_{p}=10$ particle per cells, the total number of particles leaving a cell after one time step is $n_{p} \times v_{t h} \times d t / d x \approx 0.57$. After 20 time steps, nearly all particles have left their original cell. The reference time corresponds to the standard case of 1x1x1 tile. The tests were performed on one MPI process and a single socket, on the Edison cluster at NERSC.

Notice that at each time step, the particles of each tile are advanced and then exchanged between tiles. As particles move less than one cell at each time step, the amount of particles exchanged between tiles at each time step is low for typical tiles' sizes. (The surface/volume ratio decreases with tile size.) As a consequence, particle exchanges between tiles account in practice for a very small percentage of the total PIC loop (a few percents). Our particle exchange algorithm differs from the one used in [12] in that it avoids copying data into buffers. In addition, it can be efficiently parallelized using OpenMP (details are beyond the scope for this paper and will be presented in an upcoming publication).

\subsubsection{Multi-level parallelization}

PICSAR-EM3D also includes the following high performance implementations:

(i) vectorization of deposition and gathering routines,

(ii) OpenMP parallelization for intranode parallelisms. Each OpenMP thread handles one tile. As there are much more tiles than threads in 3D, load balancing can be easily done using the SCHEDULE clause in openMP with the guided attribute,

(iii) MPI parallelization for internode parallelism,

(iv) MPI communications are overlapped with computations. For particles, this is done by treating exchanges of particles with border tiles while performing computations on particles in inner tiles,

(v) MPI-IO for fast parallel outputs.

In the remainder of this paper, we will focus on the vectorization of direct charge/current deposition routines for their simplicity and widespread use in electromagnetic PIC codes. The Esirkepov-like current deposition is not treated in this paper but the techniques used here are very general and should apply in principle to any kind of current deposition.

\section{Former CRAY vector algorithms and performance challenges on new architectures}

In the following, we focus on the direct $3 D$ charge deposition which can be presented in a more concise way than the full $3 D$ current deposition. The full $3 \mathrm{D}$ scalar/vector algorithm presented here for charge/current deposition can be found in the Fortran source file vec_deposition.F90 of the CPC Program Library archive associated to this paper. In this file, scalar charge deposition routines have the following prefix: "depose_rho_scalar_". Order is specified at the end. For instance order 1 charge scalar deposition routine is named depose_rho_scalar_1_1_1. For vector deposition routines, the prefix uses is "depose_rho_vecHVv2_". The same notation is used for current deposition routines but with the prefixes "depose_jxjyjz_scalar_"/ "depose_jxjyjz_vecHVv2_" for scalar/vector routines. 


\subsection{Scalar algorithm}

The scalar algorithm for order 1 charge deposition is detailed in listing 1 . For each particle index $i p$, this algorithm (see line 5):

(i) finds the indices $(j, k, l)$ of the cell containing the particle (lines $11-13$ ),

(ii) computes the weights of the particle at the 8 nearest vertices $w 1$ to $w 8$ (line 15-not shown here),

(iii) adds charge contribution to the eight nearest vertices $\{(j, k, l),(j+1, k, l),(j, k+1, l),(j+1, k+$ $1, l),(j, k, l+1),(j+1, k, l+1),(j, k+1, l+1),(j+1, k+1, l+1)\}$ of the current cell $(j, k, l)$ (see lines $18-25)$.

Listing 1: Scalar charge deposition routine for CIC particle shape factors

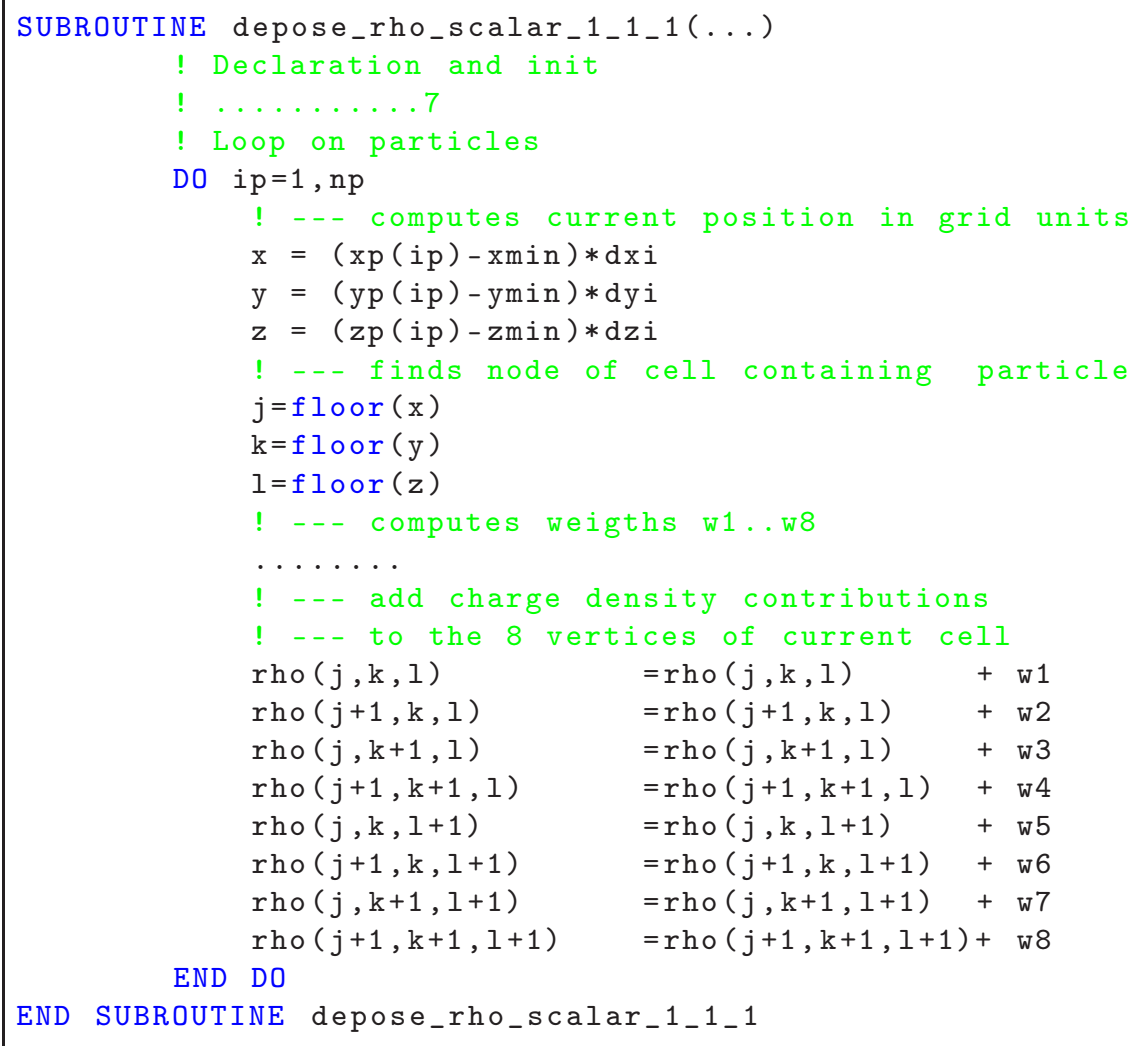

As two different particles $i p_{1}$ and $i p_{2}$ can contribute to the charge at the same grid nodes, the loop over particles (line 5) presents a dependency and is thus not vectorizable as is.

\subsection{Former vector algorithms and new architecture constraints}

Several vector algorithms have already been derived and tuned on former Cray vector machines $[3,4,5$, $6,7,13$. However, these techniques are not adapted anymore to current architectures and yield very poor results on SIMD machines that necessitate to comply with the three following constraints in order to enable vector performances:

(i) Good cache reuse. The flop/byte ratio (i.e. cache reuse) in the main loops of the PIC algorithm must be high in order to observe a speed-up with vectorization. Otherwise, if data has to be moved from memory to caches frequently, the performance gain with vectorization can become obscured by the cost of data movement. As we showed earlier, this is ensured by particle tiling in our code,

(ii) Memory alignement. Data structures in the code need to be aligned and accessed in a contiguous fashion in order to maximize performances. Modern computers read from or write to a memory address in word-sized chunks of 8 bytes (for 64 bit systems). Data alignment consists in putting the data at a memory address equal to some multiple of the word size, which increases the system's performance due to the way the CPU handles memory. SSE2, AVX and AVX-512 on x86 CPUs do require the data 


\subsection{Example: the Schwarzmeier and Hewit scheme (SH)}

\subsubsection{SH vector deposition routine}

Listing 2 details the Schwarzmeier and Hewitt (SH) deposition scheme [5] that was implemented in PICSAR-EM3D and tested on Cori supercomputer at NERSC. In this scheme, the initial loop on particles is done by blocks of lengths nblk (cf. line 5) and split in two consecutive nested loops:

- A first nested loop (line 7) that computes, for each particle $n n$ of the current block:

(i) its cell position ind0 on the mesh (line 13),

(ii) its contribution $w w(1, n n), \ldots, w w(8, n n)$ to the charge at the 8 vertices of the cell and

(iii) the indices $l l(1, n n), \ldots, l l(8, n n)$ of the 8 nearest vertices in the $1 \mathrm{D}$ density array rho (cf. lines $14-19)$.

Notice that 1D indexing is now used for rho to avoid storing three different indices for each one of the 8 vertices. The Fortran integer array $\operatorname{mof} f(1: 8)$ gives the indices of the 8 vertices with respect to the cell index ind 0 in the 1D array rho. The loop at line 7 has no dependencies and is vectorized using the portable $\$ O M P$ SIMD directive.

- A second nested loop (line 23) that adds the contribution of each one of the nblk particles to the 8 nearest vertices of their cell (line 26). As one particle adds its contribution to eight different vertices, the loop on the vertices at line 25 has no dependency and can also be vectorized using the $\$ O M P S I M D$ directive.

Usually, $n b l k$ is chosen as a multiple of the vector length. Notice that using a moderate size $n b l k$, for the blocks of particles, ensures that the temporary arrays $w w$ and $l l$ fit in cache.

The SH algorithm presented on listing 2 is fully vectorizable and gave very good performances on former Cray machines $[5,7]$. However as we show in the following section, it yields very poor performances on SIMD architectures.

Listing 2: Vector version of the charge deposition routine developed by SH for CIC particle shape factors

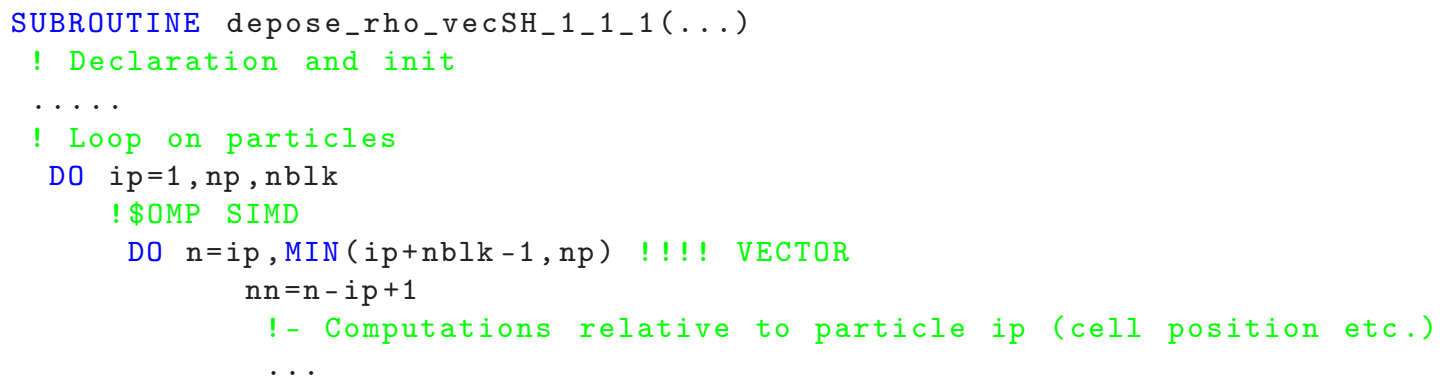






\subsubsection{Tests of the Schwarzmeier and Hewit algorithm on Cori}

The SH algorithm was tested on one socket of the Cori cluster at NERSC. This socket had one Haswell Xeon processor with the following characteristics:

(i) 16-core $\mathrm{CPU}$ at $2.3 \mathrm{GHz}$,

(ii) 256-bit wide vector unit registers (4 doubles, 8 singles) with AVX2 support,

(iii) $256 \mathrm{kB}$ L2 cache/core, 40MB shared L3 cache.

The Intel compiler was used to compile the code with option "-O3". The simulation was ran using 1 MPI process and 1 OpenMP thread per MPI process, with the following numerical parameters:

(i) $100 \times 100 \times 100$ grid points with $10 \times 10 \times 10=1000$ tiles i.e 10 tiles in each direction,

(ii) Two particle species (proton and electron) with 10 particle per cells. The particles are randomly distributed across the simulation domain. Plasma electrons have an initial thermal velocity of $\approx 0.1 c$.

The results are displayed on table 3.3.2 for order 1 scalar and $\mathrm{SH}$ routines, using two different compiler options in each case:

(i) -xCORE-AVX2 to enable vectorization,

(ii) -no-vec to disable auto-vectorization of the compiler. In this case, we also manually remove ! $\$ \mathrm{OMP}$ SIMD directives to avoid simd vectorization of loops.

\begin{tabular}{|c|c|c|c|c|}
\hline Routine & \multicolumn{2}{|c|}{ depose_rho_scalar_1_1_1 } & \multicolumn{2}{|c|}{ depose_rho_vecSH_1_1_1 } \\
\hline Compiler option & -no-vec & -xCORE-AVX2 & -no-vec & -xCORE-AVX2 \\
\hline Time/it/part & $14.6 n s$ & $14.6 n s$ & $21 n s$ & $15.9 n s$ \\
\hline
\end{tabular}

Table 3: Performance comparisons of scalar and SH vector routines.

The scalar routine takes the same time for -xCORE-AVX2 and -no-vec options because the routine is not auto-vectorizable by the compiler.

For the vector routine, we see an improvement of 30\% between -xCORE-AVX2 and -no-vec options, showing that vectorization is enabled and working in the -xCORE-AVX2 case. Nevertheless, the overall performance is poor, and the vector routine compiled with -xCORE-AVX2 is even $10 \%$ slower than the scalar routine.

By looking at the code on listing 2 and using compiler report/ assembly code generated by the Intel compiler, we found two main reasons for this poor performance: 


\section{New and portable SIMD algorithms}

In this section, we present vector algorithms that perform efficiently on SIMD architectures.

\subsection{CIC (order 1) particle shape}

\subsubsection{Algorithm}

The new vector algorithm is detailed on listing 3. Similarly to the SH routine, the main particle loop is done by blocks of $n b l k$ particles and divided in two consecutive nested loops: $(i)$ a first nested loop that computes particle weights and $(i i)$ a second one that adds the particle weights to its 8 nearest vertices.

\subsubsection{Improvements brought by the new algorithm}

The new algorithm adresses the two main bottlenecks of the $\mathrm{SH}$ algorithm with the two following new features:

1. a new data structure for $r h o$ is introduced, named rhocells, which enables memory alignement and unit-stride access when depositing charge on the 8 vertices. In rhocells, the 8-nearest vertices are stored contiguously for each cell. The array rhocells is thus of size $(8, N C E L L S)$ with $N C E L L S$ the total number of cells. The element rhocells $(1, i c e l l)$ is therefore 64 bytes-memory aligned for a given cell icell and the elements rhocells $(1: 8, i c e l l)$ entirely fit in one cache line allowing for efficient vector load/stores. The array rhocells is reduced to rho once, after the deposition is done for all particles (cf. line 46). This step is easily vectorizable (see line 48) but might not lead to optimal performances due to the non-contiguous access in rho that leads to gather-scatter instructions. Notice however that this time, this operation is proportional to the number of cells $N C E L L S$ and not to the number of particles $n p$ as it was in the case of the $\mathrm{SH}$ algorithm. The overhead is thus proportionally lower when there are more particles than cells, which is the case in many PIC simulations of interest,

2. for each particle, the 8 different weights $w w$ are now computed using a generic formula (see line $41-42$ ) that suppresses gather instructions formerly needed in the SH algorithm. This also avoids implementing non-portable efficient transpose between the first and second loop, rendering this new algorithm fully portable. 


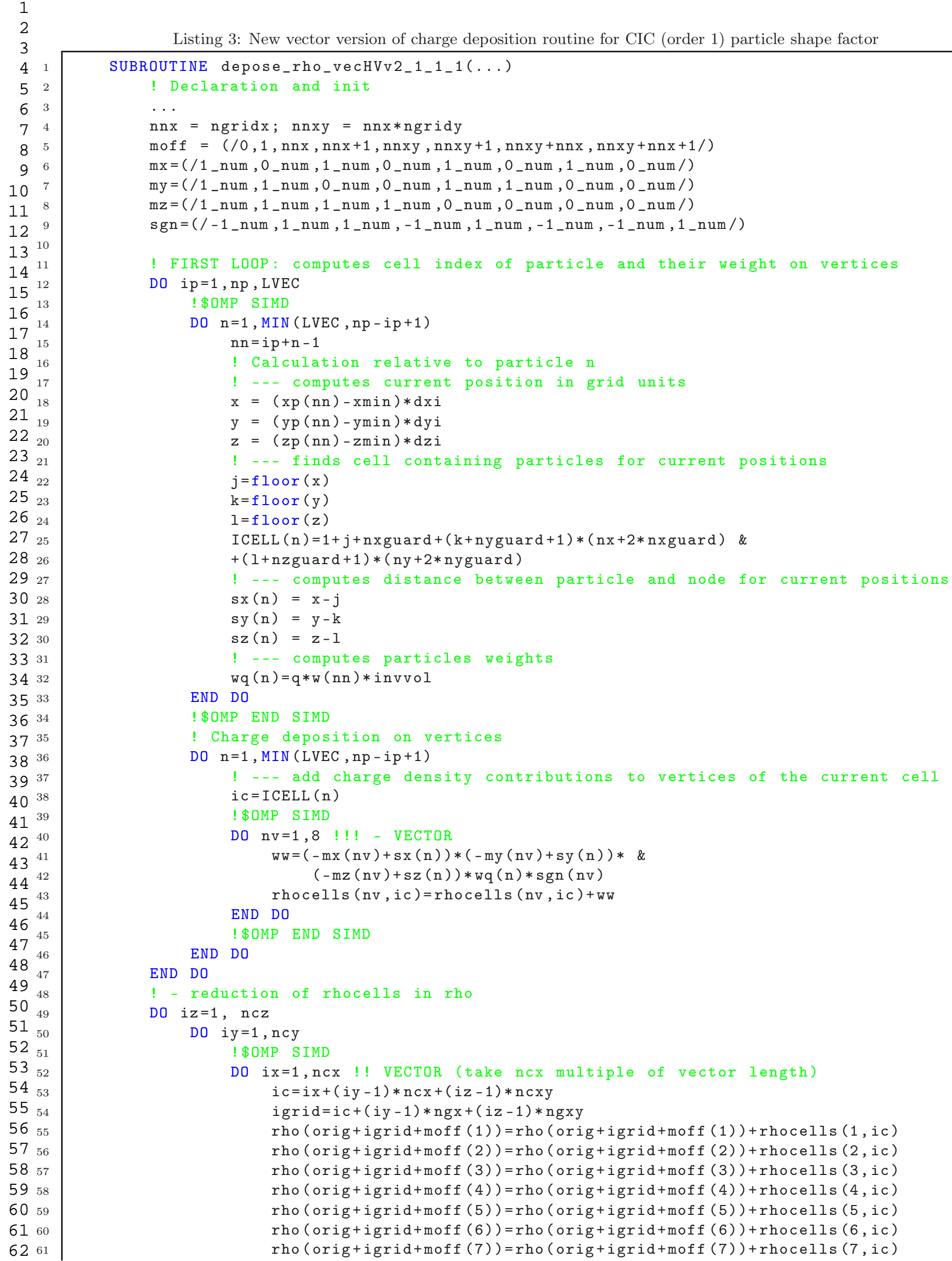

Listing 3: New vector version of charge deposition routine for CIC (order 1) particle shape factor 


\subsection{Higher particle shape factors}

Similar algorithms were derived for order 2 (TSC) and order 3 particle shape factors, and are detailed in the source file vec_deposition.F90 in the Program library. Corresponding current deposition algorithms can also be found in this file for orders 1,2 and 3 depositions. In these algorithms, we use three structures $j x c e l l s, j y c e l l s$ and $j z c e l l s$ (analogous to rhocells for the deposition of $r h o$ ) for the current components $j x$, $j y, j z$ along directions $x, y$ and $z$.

In the following, we detail the data structures used for rhocells for orders 2 and 3 particle shapes (cf. Fig. 3):

(a)

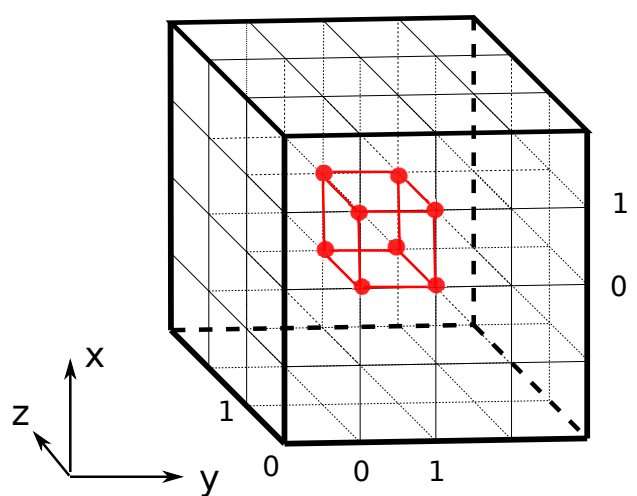

(b)

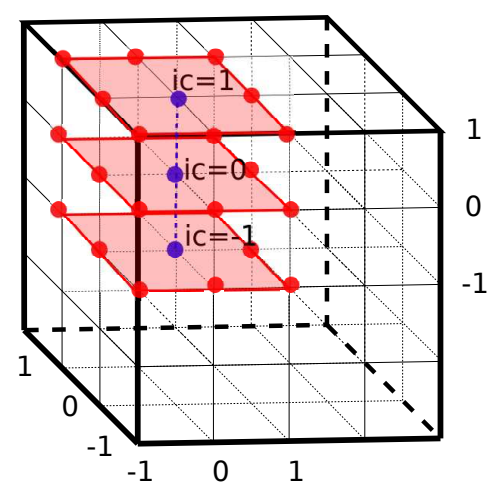

(c)

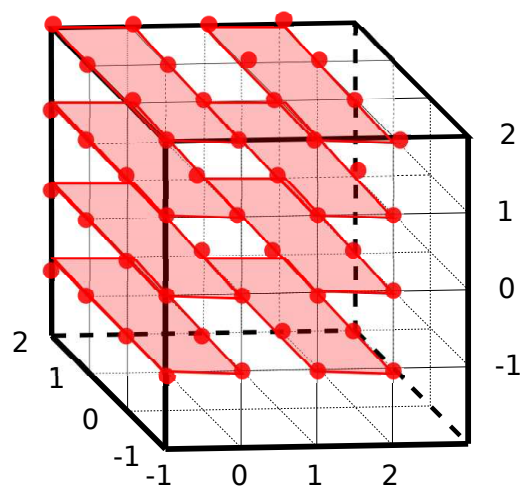

Figure 3: Data structure used for the array rhocells for different particle shape factors. In each plot, the particle that deposits charge to its nearest vertices (red/blue points) is located in the cell at position $(0,0,0)$. (a) CIC (order 1 ) particle shape factor. The particle deposits its charge to the eight nearest vertices (red points). For each cell $i c e l l=(j, k, l)$, rhocells stores the 8 nearest vertices $(j, k, l),(j+1, k, l),(j, k+1, l),(j+1, k+1, l),(j, k, l+1),(j+1, k, l+1),(j, k+1, l+1)$ and $(j+1, k+1, l+1)$ contiguously. (b) TSC (order 2) particle shape factor. The particle deposits its charge to the 27 neighboring vertices (red and blue points). For a given cell $i c e l l=(j, k, l)$ rhocells stores contiguously the 8 vertices (red points) $(j, k-1, l-1),(j, k, l-1),(j, k+1, l-1),(j, k-1, l),(j, k+1, l),(j, k-1, l+1),(j, k, l+1)$ and $(j, k+1, l+1)$. The blue points are not stored in rhocells and are treated scalarly in the algorithm. (c) QSP (order 3) particle shape factor. The particle deposits its charge to the 64 neighboring vertices (red points). For a given cell icell $=(j, k, l)$, rhocells stores contiguously the 8 vertices (delimited by red areas) $(j, k-1, l-1),(j, k, l-1),(j, k+1, l-1),(j, k+1, l-1),(j, k-1, l),(j, k, l),(j, k+1, l)$, $(j, k+1, l)$.

(i) TSC (order 2) particle shape. (cf. panel(b) of Fig. 3 and subroutine depose_rho_vecHVv2_2_2_2 in source file in source file vec_deposition.F90 in Program Library). In this case, the particles deposit their charge to the 27 neighbouring vertices. However, storing 27 contiguous vertices per cell in rhocells would not be efficient as the reduction of rhocells to rho would be much more expensive with potential cache-reuse inefficiency. Instead, while the same size for rhocells $(1: 8,1: N C E L L S)$ is used, the vertices are now grouped in a different way. The new structure for rhocells $(1: 8,1: N C E L L S)$ groups 8 points in a $(y, z)$ plane for each cell icell (see red points in red areas). For each cell, each particle adds its charge contribution to 24 points in the three planes at icell -1 , icell and icell +1 . The three remaining central points (blue points) can be either treated scalarly for 512-bits wide vector registers or vectorized for 256-bits by artificially adding a virtual point that does not contribute to any 
charge. Notice that we did not find a generic formulation for the weights $w w$ and we are therefore still performing a "gather" instruction for $w w$ in the loop on the vertice (line 2089 in vec_deposition.F90). However, this gather is performed in the $y$ and $z$ directions for the first plane of 8 points (plane $i c=-1$ on panel (b)) and is subsequently reused on the two other planes $i c=0$ and $i c=1$ (see lines 2091 to 2095 in vec_deposition.F90). Gather is thus performed only 8 times out of 24 points and thus has a limited impact on performance, as shown below in the reported test results.

(ii) QSP (order 3) particle shape. (cf. panel(c) of Fig. 3 and subroutine depose_rho_vecHVv2_3_3_3 in source file vec_deposition.F90 in Program Library). In this case, particles deposit their charge to the 64 neighbouring vertices. rhocells $(1: 8,1: N C E L L S)$ also group 8 points in a $(y, z)$ plane but differently from the TSC case (see red areas in panel (c)). For each cell, each particle adds its charge contribution to 64 points in the 8 different $(y, z)$ planes at icell $-n c x-1$, icell $-n c x$, icell $-n c x+1$, $i c e l l-n c x+2, i c e l l+n c x-1, i c e l l+n c x, i c e l l+n c x+1$ and $i c e l l+n c x+2$ where $n c x$ is the number of cells in the $x$ direction (see lines 2434 to 2450 in vec_deposition.F90). This might reduce the flop/byte ratio of the second loop when $n n x$ is large enough so that elements rhocells $(1: 8, i c e l l)$ and rhocells $(1: 8$, icell $+n n x-1)$ are not in $L 1$ cache. The vertices could have been grouped in $(y, z)$ planes of 16 points instead of 8 points but this would imply a bigger reduction loop of rhocells in rho and worst performances for a low number of particles. Notice that here again, we did not find an efficient generic formulation for the weights $w w$ and we are therefore still performing a "gather" instruction (see lines 2432 and 2442 in vec_deposition.F90). However, this gather is performed in the $y$ and $z$ directions and gathered values are subsequently reused for computing the weights at different positions in $x$ (see lines 2434 to 2440 and 2444 to 2450 in vec_deposition.F90). Gather is thus performed only 16 times out of 64 points and thus has a limited impact on performance, as shown below in the reported test results.

\section{Speed-up brought by the new vector algorithm on Cori}

The new vector algorithms were benchmarked on one node (two sockets) of the Cori machine in the same numerical conditions than the ones used in section 3.3.2 but with 2 MPI processes (one per socket) and 16 OpenMP threads per MPI process. For charge deposition, we use 10x10x10 tiles in each direction. For current deposition, we use a larger number of tiles $(12 \times 12 \times 12$ tiles in each direction) so that the three structures jxcells, jycells and jzcells (equivalent of rhocells for current deposition) fit in cache. Results are shown on Fig. 4 for charge deposition and on Fig. 5 for current deposition. Panels (a) show the time/iteration/particle (in ps for Fig. 4 and ns for Fig. 5) for scalar and vector algorithms with different particle shape factors and when there are 64 times more particles than cells. Panels (b) show the evolution of the speed-up brought by the vector algorithm over the scalar one when the number of particles per cell is varied.

Even for a low number of particles per cell (e.g panels (b) of Figs. 4 and 5), the algorithm performs well, with speed-ups of up to $\times 1.8$. When the number of particles increases performances are even better because the reduction operation of rhocells/jcells structure in regular arrays $r h o / j$ becomes more and more negligible relatively to particle loops. For 64 times more particles than cells, performances for charge deposition now reach $\times 2.7$ with an order 1 particle shape factor. Order 3 charge/current deposition performs less efficiently than orders 1 and 2, because as we described in the previous section, the structure we chose for rhocells/jcells decreases the flop/byte ratio of the loop on vertices compared to orders 1 and 2 . In the case of simulations using a lot of particles, for which the reduction of rhocells in rho is negligible, one might consider grouping vertices in rhocells by groups of 16 instead of 8 for order 3 deposition in order to increase the flop/byte ratio in loop on vertices. Notice finally that as we vectorize on vertices, there is no performance bottleneck related to a possibly inhomogeneous distribution of particles on the simulation domain.

If speed-ups from particle tiling $(3 \times)$ and from vectorization (up to $2.7 \times$ for charge deposition at order 1) are combined, we can gain a cumulated speed-up of up to $8 \times$ for deposition routines. 


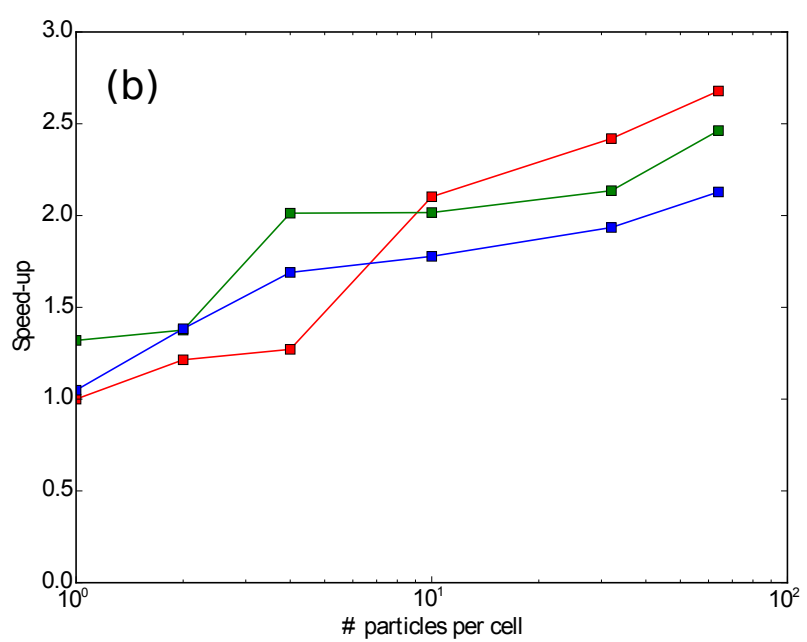

Figure 4: Speed-up brought by vectorization with the new charge deposition algorithm on Cori. (a) Each bar plot shows the time/it/part in $p s$ for different particle shape orders 1 to 3 . Benchmarks were done with 64 times more particles than cells. (b) Evolution with the number of particles per cell of the speed-up gain brought by vector routines compared to scalar routines. Red/Green/Blue curves are evolution of the speed-up gain for orders $1 / 2 / 3$ charge deposition respectively.


Figure 5: Speed-up brought by vectorization with the new current deposition algorithm on Cori. (a) Each bar plot shows the time/it/part in ps for different particle shape orders 1 to 3. Benchmarks were done with 64 times more particles than cells. (b) Evolution with the number of particles per cell of the speed-up gain brought by vector routines compared to scalar routines. Red/Green/Blue curves are evolution of the speed-up gain for orders 1/2/3 current deposition respectively.

\section{Conclusion and prospects}

A new method is presented that allows for efficient vectorization of the standard charge/current deposition routines on current SIMD architectures, leading to efficient deposition algorithms for shape factors of orders 1, 2 and 3. This method uses a new data structure for grid arrays (charge/currents) ensuring data alignement and contiguity in memory which are essential to avoid many gather/scatter operations that can significantly hinder vector performances on modern architectures. The algorithms can be used on current multi-core architectures (with up to AVX2 support) as well as on future many-core Intel $K N L$ processors that will support $A V X-512$. Further tests on KNL will be performed as the processor becomes available.

This work presents deposition routines that are fully portable and only use the \$OMP SIMD directives that are provided by OpenMP 4.0. Efficient vectorization of the charge conserving current deposition from 
Esirkepov is being investigated, and will be detailed in future work.

\section{Acknowledgement}

We thank Karthik Raman from Intel inc for useful discussions on the optimization of the vector routines. This work was supported by the European Commission through the Marie Skłowdoska-Curie actions (Marie Curie IOF fellowship PICSSAR grant number 624543) as well as by the Director, Office of Science, Office of High Energy Physics, U.S. Dept. of Energy under Contract No. DE-AC02-05CH11231, the US-DOE SciDAC program ComPASS, and the US-DOE program CAMPA. This research used resources of the National Energy Research Scientific Computing Center, a DOE Office of Science User Facility supported by the Office of Science of the U.S. Department of Energy under Contract No. DE-AC02-05CH11231.

This document was prepared as an account of work sponsored in part by the United States Government. While this document is believed to contain correct information, neither the United States Government nor any agency thereof, nor The Regents of the University of California, nor any of their employees, nor the authors makes any warranty, express or implied, or assumes any legal responsibility for the accuracy, completeness, or usefulness of any information, apparatus, product, or process disclosed, or represents that its use would not infringe privately owned rights. Reference herein to any specific commercial product, process, or service by its trade name, trademark, manufacturer, or otherwise, does not necessarily constitute or imply its endorsement, recommendation, or favoring by the United States Government or any agency thereof, or The Regents of the University of California. The views and opinions of authors expressed herein do not necessarily state or reflect those of the United States Government or any agency thereof or The Regents of the University of California. 


\section{References}

[1] Birdsall and Langdon, Plasma Physics via computer simulation, 15-5

[2] P. Kogge, K. Bergman, S. Borkar, D. Campbell, W. Carlson, W. Dally, M. Denneau, P. Fran- zon, W. Harrod, K. Hill, J. Hiller, S. Karp, S. Keckler, D. Klein, R. Lucas, M. Richards, A. Scarpelli, S. Scott, A. Snavely, T. Sterling, R. S. Williams, and K. Yelick. Exascale computing study: Technology challenges in achieving exascale systems. Technical report, DARPA, 2008

[3] A. Nishiguchi, S. Orii, T. Yabe, Journal of Computational Physics, 61 (1985) 519

[4] E. J. Horowitz, Journal of Computational Physics, 68 (1987) 56

[5] J. L. Schwarzmeier, T. G. Hewitt,Proceedings, 12th conf. on numerical simulation of plasmas, 1987, San Francisco.

[6] A. Heron, J.C. Adam, Journal of Computational Physics, 85 (1989) 284-301

[7] G. Paruolo, Journal of Computational Physics, 89 (1990) 462-482

[8] R.A. Fonseca, J.Vieira, F. Fiuza, A. Davidson, F. S. Tsung, W. B. Mori and L. O. Siva ArXiv, http://arxiv.org/pdf/1310.0930v1.pdf (2013)

[9] http://www.nersc.gov

[10] http://warp.lbl.gov

[11] T. Esirkepov, Computer Physics Communications, 135 (2001) 144-153

[12] Viktor K. Decyk, Tajendra V. Singh, Computer Physics Communications, 185 (2014) 708-719

[13] David V. Anderson, Dan E. Shumaker, Computer Physics Communications, 87 (1995) 16-34 